\title{
Effects of provision and receipt of social support on adjustment to laparoscopic radical prostatectomy
}

\author{
URTE SCHOLZ ${ }^{1}$, NINA KNOLL ${ }^{2}$, JAN ROIGAS ${ }^{3}$, \& OLIVER GRALLA ${ }^{4}$ \\ ${ }^{1}$ Department of Psychology, Social and Health Psychology, University of Zurich, Zurich, Switzerland; \\ ${ }^{2}$ Institute of Medical Psychology, Charité-Universitätsmedizin Berlin, Berlin, Germany; ${ }^{3}$ Department \\ of Urology, Vivantes Hospital Am Urban, Berlin, Germany $\mathcal{F}^{4}$ Department of Urology, University \\ Hospital Cologne, Cologne, Germany
}

\begin{abstract}
Radical prostatectomy has a negative impact on prostate cancer patients' health-related quality of life (HRQoL). Social support may help patients adjust to their situation after surgery. We investigated patients' accounts of received social support as well as their spouses' accounts of provided social support as predictors of patients' HRQoL 6 months after surgery. Moreover, we tested whether patients with lower HRQoL at 2 weeks after surgery had the most benefit from spousal support. A total of 77 patients $(M=61.57$ years, $S D=6.03)$ and their spouses $(M=58.46$ years, $S D=7.52)$ completed questionnaires at 2 weeks and 6 months after laparoscopic radical prostatectomy. Received social support, spousal provided social support, and HRQoL were assessed 2 weeks after surgery, and HRQoL was assessed again 6 months later. Patients with lower HRQoL at 2 weeks after surgery benefited more from the receipt and their partners' provision of support in terms of HRQoL 6 months later than patients with a higher HRQoL at the beginning. Assessing patients' HRQoL might be one method to identify those individuals who are in special need of support from their spouses.
\end{abstract}

Keywords: Social support receipt, social support provision, cancer, prostatectomy, health-related quality of life

Prostate cancer is one of the most frequently diagnosed types of cancer in men in the United States. In 2003, 164.9 men out of 100,000 were diagnosed with prostate cancer (Ries et al., 2006). The same applies to European countries. In Germany, prostate cancer is the second most frequent cause of cancer deaths among men (German Federal Statistical Office, 2006).

Radical prostatectomy is one standard treatment for patients with a localized prostate carcinoma (Eton \& Lepore, 2002). Although only small incisions are needed to remove the prostate by means of laparoscopic techniques (laparoscopic radical prostatectomy (LRPE)), and surgical trauma is kept at a minimum (Roumeguere et al., 2003; Türk, Deger, Winkelmann, Schönberger, \& Loening, 2001), patients are still faced with a number of stressors commonly associated with the aftermath of radical prostatectomy. Such stressors

Correspondence: Urte Scholz, Department of Psychology, Social and Health Psychology, University of Zurich, Binzmuehlestr. 14/Box 14, CH-8050 Zürich, Switzerland. Tel: +41 44 6357255. Fax: +41 44635 7259. E-mail: urte.scholz@psychologie.uzh.ch 
are postoperative pain, incontinence, and sexual dysfunctions (Basillote, Ahlering, Skarecky, Lee, \& Clayman, 2004; Roumeguere et al., 2003; Türk et al., 2001).

\section{Health-Related Quality of Life (HRQoL) in Prostate Cancer Patients}

Health-related quality of life (HRQoL) is conceptualized as the physical, mental, and social effects illness has on daily life, and the impact of these effects on levels of subjective wellbeing, satisfaction, and self-esteem (Bowling, 1991). Diagnosis and treatment of prostate cancer have been shown to have an impact on patients' HRQoL (for an overview, see Eton \& Lepore, 2002). Visser et al. (2003) found a decrease in HRQoL in prostate cancer patients 3 months after treatment compared to patients with benign prostate hyperplasia. In addition, Lubeck and colleagues (1999) found that patients undergoing radical prostatectomy had substantially reduced HRQoL 1 year after surgery. These effects, however, seem to vanish over time. In the same study, patients reported an increase in HRQoL 2 years after surgery (Lubeck et al., 1999). The results of Litwin and colleagues' study (Litwin, McGuigan, Shpall, \& Dhanani, 1999) indicated that prostatectomy patients recover in terms of HRQoL within 2 years following the operation. On average, patients receiving radical prostatectomy adjust relatively well to the treatment and related consequences (Braslis, Santa-Cruz, Brickman, \& Soloway, 1995).

Marital status seems to play an important role during recovery from severe illnesses and treatment-related side effects. For example, 1 year following radical prostatectomy, unmarried men reported slower and less pronounced increases in HRQoL when compared to married men (Litwin et al., 1999). Being married seems to be especially beneficial for men with regard to several domains of well-being and HRQoL (Coombs, 1991). This effect is mainly attributed to social support processes that take place between married couples (e.g., Cohen \& Wills, 1985).

\section{Social Support and HRQoL in Prostate Cancer Patients}

Several types of social support should be differentiated, such as perceived social support and received and provided social support (Schwarzer, Knoll, \& Rieckmann, 2003). Perceived social support comprises the potential availability of social support from the social network (Sarason, Pierce, \& Sarason, 1990). Perceived social support is known to be relatively stable, and it depends only little on actual support transactions (e.g., Sarason, Sarason, \& Shearin, 1986). In contrast, received social support refers to recipients' reports of actual support transactions. This requires that another person (e.g., the spouse) has actually provided social support (such as offering information; for an overview, see Schwarzer et al., 2003).

Numerous studies have demonstrated the positive effects of social support on psychological and physical adjustment in patients with different types of cancer (Helgeson, 2003; Parker, Baile, DeMoor, \& Cohen, 2003; Schulz \& Schwarzer, 2004) as well as in prostate cancer patients (Ptacek, Pierce, Ptacek, \& Nogel, 1999; Roberts, Lepore, \& Helgeson, 2006; Visser et al., 2003). However, closer scrutiny reveals that most of these studies investigated the effects of perceived social support on HRQoL (e.g., Roberts et al., 2006). One exception is the study by Boehmer and colleagues on the effects of received social support on HRQoL in different cancer patients (Boehmer, Luszczynska, \& Schwarzer, 2007). The authors found a positive effect of received social support, measured 1 month after cancer surgery, on emotional well-being 5 months later. This effect emerged 
while controlling for self-efficacy, thus emphasizing the importance of social resources in addition to personal resources. Moreover, the role of a spouse's behavior and characteristics on a patient's adjustment to diagnosis and treatment of prostate cancer has received comparatively little attention so far (Campbell et al., 2004; Eton, Lepore, \& Helgeson, 2005; Gray, Fitch, Phillips, Labreque, \& Fergus, 2000; Maliski, Heilemann, \& McCorkle, 2002).

The studies described above examined main effects of social support on HRQoL. The Stress Buffering Hypothesis (Cohen \& Wills, 1985) provides yet another important perspective on the interplay between support processes and distress. The Stress Buffering Hypothesis postulates that there might be differences in the effectiveness of social support, depending on the stress level of a person: Individuals with high levels of distress might benefit more from social support (Cohen \& Wills, 1985). These benefits might occur, for example, by helping the afflicted person to adjust his or her response to the threat by reappraising the situation, ceasing maladaptive responses, or facilitating functional coping efforts. Evidence for this buffering hypothesis was also found in cancer patients (e.g., Koopman, Hermanson, Diamond, Angell, \& Spiegel, 1998). In this latter study with women suffering from breast cancer, social support was related to lower distress only for women who had experienced greater life stress (Koopman et al., 1998).

In the case of prostate cancer, most studies demonstrated that 2 years after radical prostatectomy patients' HRQoL is largely restored. There is, however, considerable variance in prostate cancer patients' HRQoL within the first year after treatment (Eton \& Lepore, 2002). It has not yet been tested whether this restoration of HRQoL varies over time depending on the initial level of HRQoL. Resembling the idea of the Stress Buffering Hypothesis (Cohen \& Wills, 1985), those individuals with a lower HRQoL at baseline might benefit more from social support than those with a higher HRQoL at baseline.

\section{Aim of the Present Study}

In our study, we first sought to investigate whether the provision of support by spouses and the receipt of this support by the patients are beneficial in terms of patients' reported HRQoL (physical functioning and mental component). Moreover, in line with the idea of the Stress Buffering Hypothesis (Cohen \& Wills, 1985), we investigated whether there is evidence for the moderating role of baseline HRQoL in that the beneficial effects of providing and receiving social support are accentuated in patients who begin with a lower HRQoL.

\section{Method}

\section{Sample and Design}

Our study was part of a larger project on prostatectomy patients' and their spouses' perioperative stress and support processes (see Knoll, Burkert, Rosemeier, Roigas, \& Gralla, 2007). Consecutive patients and their spouses were contacted by study personnel upon admission to the urology ward at the hospital. Couples agreeing to participate were given information material, informed consent forms, and presurgery questionnaires. Two weeks (Time 1 [T1]) and 6 months (Time 2 [T2]) after surgery, couples received additional questionnaires via mail. The sample at T1 consisted of 77 patients receiving LRPE and their spouses. Six months later, at T2, 63 couples completed the second set of questionnaires $(81.8 \%)$. 
Mean age of patients was $M=61.57$ years, $S D=6.03$; range $=44-73$, and mean age of partners was $M=58.46$ years, $S D=7.52$; range $=38-73$. Most couples, $n=67$, were married, nine were in a stable relationship, and one patient was in a new relationship with his partner; $85.7 \%$ of the couples had children. All patients and $79.2 \%$ of the spouses provided data on their education. About half of the patients $(48.1 \%)$ and about half of the spouses with valid data (57.4\%) reported 9 to 10 years of schooling, and $49.4 \%$ of patients and $41 \%$ of spouses reported 12 to 13 years of schooling (the remaining participants did not give details on their education). About half of the patients $(58.9 \%)$ were retired.

All patients underwent LRPE. After surgery, patients stayed at the hospital for a mean of 5.94 days, $S D=2.15$. Four patients experienced gastrointestinal, pneumonal, or cardiovascular complications after surgery, and one patient had to be transferred to the intensive care unit. For five patients, data on postoperative complications were not available.

Dropout analyses revealed that patients who did not complete the T2 questionnaires did not differ from patients who continued participation in terms of age, years of schooling, marital status, T1 mental and physical HRQoL, or T1 received social support. Spouses who failed to return the questionnaires at $\mathrm{T} 2$ did not differ from those who returned the questionnaires in terms of years of schooling, marital status, or provided social support, but they were significantly younger, $F(1,59)=5.97, p<.01$.

\section{Measures}

All item examples are translations from German. Means and standard deviations of measures are reported in Table I.

Social Support. Patients' received social support was assessed 2 weeks after surgery (T1) using two items of each of the two subscales received emotional support and received instrumental support from the Berlin Social Support Scales (BSSS; Schulz \& Schwarzer, 2003). Received instrumental and emotional support items were averaged to one score. Patients reported on the social support received during the past week. Items were rated on four-point Likert-type scales ranging from $0=$ does not apply at all to $3=$ applies exactly. Item examples are: "My partner made me feel that I can rely completely on her" (emotional) and "My partner took care of things I could not manage on my own" (instrumental). Internal consistency for the four-item measure was Cronbach's alpha $=.67$.

Spouses' provided social support was also assessed at 2 weeks after surgery (T1) using two items of each of the two subscales, provided emotional support and provided instrumental

Table I. Zero-order correlations, and means, and standard deviations $(S D)$ of core variables of the study.

\begin{tabular}{|c|c|c|c|c|c|c|c|c|c|c|}
\hline & 1 & 2 & 3 & 4 & 5 & 6 & 7 & 8 & Mean & $S D$ \\
\hline 1. T1 spouses' provided social support & 1 & & & & & & & & 2.74 & .43 \\
\hline 2. T1 patients' received social support & $.44^{\star \star}$ & 1 & & & & & & & 2.66 & .47 \\
\hline 3. T1 patients' mental HRQoL & .19 & -.02 & 1 & & & & & & 50.52 & 12.41 \\
\hline 4. T2 patients' mental HRQoL & .16 & .10 & $.55^{\star \star}$ & 1 & & & & & 53.87 & 10.87 \\
\hline 5. T1 patients' physical HRQoL & .17 & .05 & .17 & .06 & 1 & & & & 37.80 & 8.55 \\
\hline 6 T2 patients' physical HRQoL & .21 & $.33^{\star \star}$ & $.31^{\star \star}$ & $.24^{\star}$ & $.40^{\star \star}$ & 1 & & & 48.77 & 8.81 \\
\hline 7. Patients' age & .17 & .07 & -.03 & .07 & .08 & -.05 & 1 & & 61.57 & 5.99 \\
\hline 8. Spouses' age & .07 & -.10 & -.11 & -.03 & .05 & -.20 & $.72^{\star \star}$ & 1 & 58.98 & 7.67 \\
\hline
\end{tabular}

Note. $N=77$ couples; $\mathrm{T} 1=$ Time 1,2 weeks after surgery; T2 = Time 2,6 months after surgery. HRQoL $=$ healthrelated quality of life.

${ }^{\star} p<.05,{ }^{\star \star} p<.01$. 
support, from the BSSS (Schulz \& Schwarzer, 2003). Again, provided instrumental and emotional support items were averaged to one score. Spouses reported on the social support provided during the past week. Items were rated on four-point Likert-type scales ranging from $0=$ does not apply at all to 3 =applies exactly. Item examples are: "I assured him that he can rely completely on me" (emotional) and "I took care of things he could not manage on his own" (instrumental). Internal consistency was Cronbach's alpha $=.78$.

HRQoL was assessed using the SF-12 (Jenkinson et al., 1997; German version by Bullinger \& Kirchberger, 1998). The SF-12 is a 12-item measure that assesses general HRQoL by means of two scales: mental HRQoL and physical HRQoL. Patients were asked to rate their mental and physical functioning during the past week (e.g., "During the past week, how much did pain interfere with your normal work (including work both outside the home and housework)?"). Response categories varied, depending on the items, from yes/no to a six-point Likert scale ranging from $1=$ all the time to $6=$ none of the time. Both subscales ranged from 0 to 100 , with higher scores indicating higher HRQoL.

\section{Data Analyses}

The problem of missing data occurs in most longitudinal studies. Complete (i.e., listwise) deletion of persons with missing values is only appropriate when the missing pattern meets the assumption of missing completely at random (MCAR; Little \& Rubin, 2002). MCAR means that the dropout group is a random subsample of the entire sample without any systematic association between missings and observed values. As dropout analyses of the present study have shown that the missing pattern is at best missing at random (MAR), which means that the probability of missing values on any variable is not related to its particular value, but depends on some other variable, listwise deletion would not be the appropriate method for handling missing data. Therefore, missing values were estimated using the Multiple-Imputation (MI) method (Schafer \& Graham, 2002) employing NORM 2.03 (Schafer, 1999). MI is a Monte Carlo technique which takes the missing-data uncertainty into account by generating multiple values for one missing observation in the form of generating multiple datasets (Barnard, Rubin, \& Schenker, 2001). Missing values for one person are imputed by using the observed values from this person and adding "random noise" (Schafer \& Graham, 2002). It is beneficial to use a "rich" imputation model, that is, to include all variables that are associated with the focal variables that comprise missings. Doing so provides most valid estimates for the missing values. When interaction terms are included in the final analyses, these interaction terms also need to be included in the imputation model (Schafer \& Olsen, 1998). This was done in the present study.

After having generated the imputed datasets, each of the generated datasets is analyzed separately using a standard statistical package (in our case Mplus 4.1; Muthén \& Muthén, 1998-2006). The results are then integrated following a method suggested by Rubin (1987) to obtain overall estimates and correct standard errors. This is automatically done in Mplus 4.1. Unlike alternative single imputation methods, $\mathrm{MI}$ has the advantage of reflecting the uncertainty of missing data by the between-imputation variance (Schafer \& Graham, 2002).

MI does not make a difference between different kinds of variables, thus it is not important whether the highest (or lowest) amount of missingness is present in the dependent variable or the independent variables. As an illustration, see the example used in Schafer and Olsen's (1998) study in which the dependent variable (DV) had the highest amount of missingness (i.e., $45 \%$ ) among all variables. 
In terms of number of generated datasets, Rubin (1987) demonstrated that already a small number of datasets (e.g., three to four) is efficient with regard to the estimates when the missing rate is below $30 \%$. With increasing rate of missingness, however, more datasets are advantageous. For the present study, 10 datasets were generated. All analyses were conducted using all 10 imputed data files. Subsequently, estimates were integrated using Mplus 4.1 (Muthén \& Muthén, 1998-2006).

In addition to bivariate correlations, multiple regression analyses were conducted to investigate interaction effects. To avoid problems with multicollinearity, variables that built the interaction term were centered (Aiken \& West, 1991). To display the interaction effects, low and high values of mental or physical HRQoL at T1 as the continuous moderators were generated by adding or subtracting one standard deviation from the centered mean of the moderators (Aiken \& West, 1991).

In order to test whether the regression slopes were significant, we ran simple slope analyses (Aiken \& West, 1991). In addition, we applied the Johnson-Neyman technique for testing the region of significance (Preacher, Curran, \& Bauer, 2006). The traditional simple slope analysis approach suggested by Aiken and West (1991) has the disadvantage of picking arbitrary values of the moderator (e.g., $1 S D$ above and $1 S D$ below the mean) in order to test for significance. The Johnson-Neyman technique in contrast results in specific values of the moderator that mark the boundaries of the "region of significance." The region of significance defines all values of the moderator (e.g., HRQoL in the present study) at which the association between the criterion (e.g., HRQoL 6 months after surgery) and the predictor (e.g., received social support) is significantly different from zero at a certain alpha level (Preacher et al., 2006). As was the case in our study, the regression of the criterion on the predictor is often significant at values below the lower and above the upper boundaries of the region of significance. For the present study, an alpha level of $p=.05$ was chosen.

\section{Results}

\section{Descriptive Findings}

Table I displays means and standard deviations as well as bivariate correlations of spouses' provided social support, patients' received social support, mental HRQoL at T1 and T2, physical HRQoL at T1 and T2, and patients' and spouses' ages. Moreover, retest stabilities of mental and physical HRQoL are included. Spouses' provided social support and patients' received social support were significantly correlated at a medium effect size, which is within the range reported in the literature (Antonucci, 2001). As can be seen in Table I, spouses' provided social support was not bivariately related to any of the HRQoL measures. Patients' received social support was only significantly related to physical HRQoL at T2. Mental and physical HRQoL showed medium retest stabilities from 2 weeks to 6 months after surgery. Patients' age and spouses' age were unrelated to the investigated outcomes and were thus not included in the final models.

\section{Regression Analyses}

To examine the hypothesized interaction effect between mental HRQoL at T1 and patients' received social support on mental HRQoL at T2, a hierarchical regression analysis was conducted. The first step comprised mental HRQoL and patients' received social support at T1. In addition, physical HRQoL at T1 was included to control for common variance in 
both measures of HRQoL. In a second step, the interaction term between mental HRQoL and patients' received social support was added. Mental HRQoL and the interaction term emerged as the only significant predictors of mental HRQoL 6 months after surgery (T2). The results are displayed in Column 1 of Table II. Overall, $41 \%$ of variance could be accounted for, including an additional 9\% explained variance due to entering the interaction term in the second step of the analysis. The moderation effect of mental HRQoL and patients' received social support at T1 unfolds as displayed in Figure 1, upper panel.

Simple slope analysis (Aiken \& West, 1991), choosing $1 S D$ above and $1 S D$ below the mean as values of the moderator, demonstrated that only for low mental HRQoL at T1 did the slope prove to be different from zero, $t=2.85, p<.01$, but not for high HRQoL at T1, $t=-1.03, p=.31$. As $1 S D$ below and $1 S D$ above the centered mean as indicators for low and high mental HRQoL at $\mathrm{T} 1$ are arbitrary values, we further verified these results by testing the region of significance applying the Johnson-Neyman technique. By using this technique, it is possible to find out within which range of values of mental HRQoL at T1 the association between $\mathrm{T} 1$ received social support and T2 mental HRQoL was significantly different from zero. The analysis of the region of significance showed that the values of $T 1$ mental $H R Q o L$ at which the association between $T 1$ received social support and T2 mental HRQoL was significant were lower than $-6.41, p<.05$, and higher than 26.13, $p<.05$. As the minimum of the mean centered mental HRQoL was -36.43 and the maximum was 20.56 , only the simple slope for the individuals who reported a T1 HRQoL lower than -6.41 was significantly different from zero. Thus, only patients with a relatively low mental HRQoL at 2 weeks after surgery benefited from receiving social support from their spouse in terms of their mental HRQoL 6 months after surgery. In contrast, patients who started with a relatively high mental HRQoL did not benefit from receiving social support in terms of their mental HRQoL 6 months after surgery.

The second analysis examined the interaction between spouses' provided social support and mental HRQoL at T1 on mental HRQoL 6 months after the operation (T2). Here again, mental HRQoL and physical HRQoL as well as spouses' provided social support at T1 were included in the first step of the analysis. The interaction term was entered in the second step. A similar picture as in the previous analysis emerged: T1 mental HRQoL was a significant predictor of T2 mental HRQoL, whereas T1 physical HRQoL and spouses' provided social support were not. Moreover, a significant moderation effect emerged (see Table II). Predictors of the first step explained a total of $31 \%$ variance in mental HRQoL at $\mathrm{T} 2$. The interaction term added another significant $8 \%$ of explained variance in the criterion. Figure 1, lower panel, displays the moderation.

Simple slope analyses resulted in non-significant results for the two arbitrarily chosen values for low, $t=1.44, p>.10$, and high, $t=1.68, p>.10$, provided social support at $\mathrm{T} 1$. When using the Johnson-Neyman technique, however, a different picture emerged. Simple slopes were significant outside the following bounds of the region of significance: -16.60 , $p<.05$, and $17.90, p<.05$. As minimum and maximum of the mean centered mental HRQoL at T1 was -36.43 and 20.56 , respectively, the association between $\mathrm{T} 2$ mental HRQoL and provided social support at T1 was only significant for individuals who reported a T1 HRQoL lower than -16.60 and for those with an initial level of HRQoL higher than 17.90. Thus, again, only those patients with a relatively low mental HRQoL at 2 weeks after surgery benefited from provided social support by their spouses. Those participants who began with a rather high mental HRQoL, however, seemed to experience negative effects from their spouses' support provision. 
Table II. Coefficients of regression analyses (step 2).

\begin{tabular}{|c|c|c|c|c|c|}
\hline & \multicolumn{5}{|c|}{ Dependent variables } \\
\hline & $\begin{array}{l}\text { T2 mental } \\
\text { HRQoL }\end{array}$ & $\begin{array}{l}\text { T2 mental } \\
\text { HRQoL }\end{array}$ & $\begin{array}{l}\text { T2 mental } \\
\text { HRQoL }\end{array}$ & $\begin{array}{l}\text { T2 physical } \\
\text { HRQoL }\end{array}$ & $\begin{array}{l}\text { T2 physical } \\
\text { HRQoL }\end{array}$ \\
\hline Predictor variables & $\beta(b, S E b)$ & $\beta(b, S E b)$ & $\beta(b, S E b)$ & $\beta(b, S E b)$ & $\beta(b, S E b)$ \\
\hline T1 mental HRQoL & $.49(.43 ; .10)^{\star \star}$ & $.47(.41 ; .10)^{\star \star}$ & $.52(.45 ; .10)^{\star \star}$ & $.26(.19 ; .07)^{\star}$ & $.22(.16 ; .09)^{\#}$ \\
\hline T1 physical HRQoL & $-.05(-.07 ; .14)$ & $-.05(-.07 ; .14)$ & $-.03(-.05 ; .14)$ & $.34(.35 ; .10)^{\star \star}$ & $.35(.36 ; .12)^{\star \star}$ \\
\hline T1 received social support & $.07(1.59 ; 2.37)$ & & $.12(2.69 ; 3.12)$ & $.26(4.85 ; 2.33)^{\star}$ & \\
\hline T1 provided social support & & $-.05(-1.16 ; 3.43)$ & $-.09(-2.36 ; 3.90)$ & & $.10(2.07 ; 2.78)$ \\
\hline $\mathrm{T} 1$ received social support $\times \mathrm{T} 1$ mental HRQoL & $-.30(-.37 ; .14)^{\star \star}$ & & $-.39(-.47 ; .29) \#$ & & \\
\hline $\mathrm{T} 1$ provided social support $\times \mathrm{T} 1$ mental HRQ $\mathrm{OL}$ & & $-.29(-.39 ; .16)^{\star}$ & $.07(.10 ; .33)$ & & \\
\hline $\mathrm{T} 1$ received social support $\times \mathrm{T} 1$ physical HRQoL & & & & $-.20(-.50 ; .24)^{\star}$ & \\
\hline $\mathrm{T} 1$ provided social support $\times \mathrm{T} 1$ physical HRQ $\circ \mathrm{L}$ & & & & & $-.05(-.15 ; .37)$ \\
\hline$R^{2}$ total & $.41^{\star \star}$ & $.39 \star \star$ & $.43^{\star \star}$ & $.36^{\star \star}$ & $.24^{\star \star}$ \\
\hline$R^{2}$ change & $.09^{\star \star}$ & $.08^{\star}$ & $.10^{\star}$ & $.04^{\star}$ & .01 \\
\hline
\end{tabular}

Note. $N=77$ couples. The results displayed are the second steps of hierarchical regression analyses. $R^{2}$ change is the amount of additional variance explained by including the interaction terms in the second step. T1 $=$ Time 1,2 weeks after surgery; T2 = Time 2,6 months after surgery. HRQoL =health-related quality of life.

$\#_{p}<.10, \star_{p}<.05,{ }^{\star} p<<.01$. 

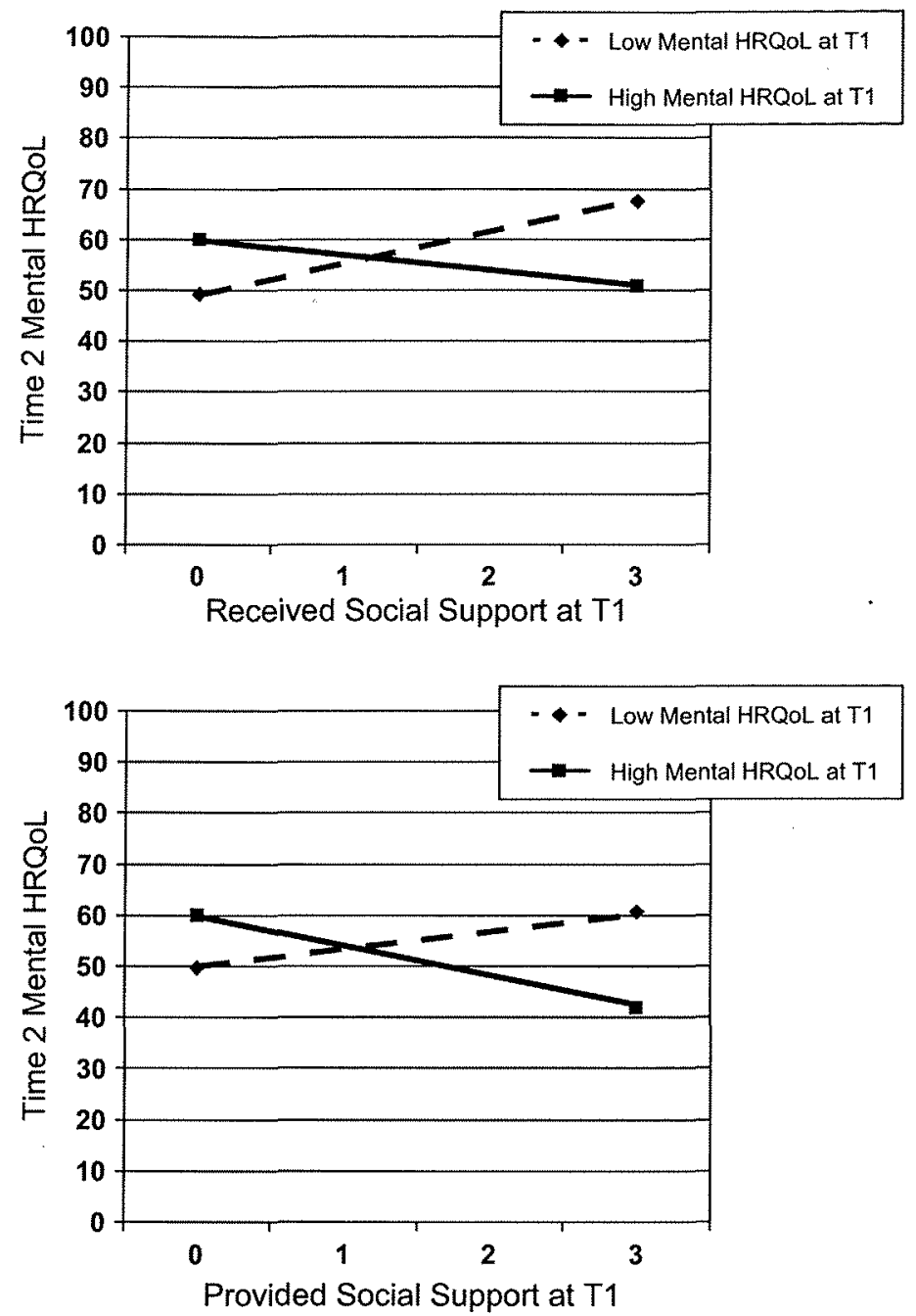

Figure 1. Upper Panel: Interaction effect between mental HRQoL 2 weeks after surgery and patients' received social support on T2 mental HRQoL. Lower Panel: Interaction effect between mental HRQoL 2 weeks after surgery and spouses' provided social support on T2 mental HRQoL.

Note. Upper Panel: Only the slope for low initial HRQoL reached statistical significance at $p=.05$. Lower Panel: Slopes for low initial HRQoL and for those with highest values in HRQoL reached statistical significance. $\mathrm{T} 1=$ Time 1, 2 weeks after surgery; T2 =Time 2, 6 months after surgery. HRQoL = health-related quality of life.

To investigate this unexpected finding more thoroughly, we conducted an analysis including both patients' received social support and spouses' provided social support as well as both interaction terms with mental HRQoL at 2 weeks after surgery as predictors of later changes in mental HRQoL. The results demonstrate that the formerly significant effect of the interaction term between mental HRQoL and spouses' provided social support was fully accounted for by the interaction term between mental HRQoL and patients' received social support (see Table II).

In the next regression analysis, T2 physical HRQoL was predicted by including $\mathrm{T} 1$ physical HRQoL, T1 patients' received social support, and T1 mental HRQoL in the first step. In a second step, the interaction between physical HRQoL and patients' received social support was entered. The analysis resulted in significant effects of T1 physical and mental HRQoL, and a significant effect of the interaction term on physical HRQoL at 6 months after surgery (see Table II). The moderation effect is displayed in Figure 2. 


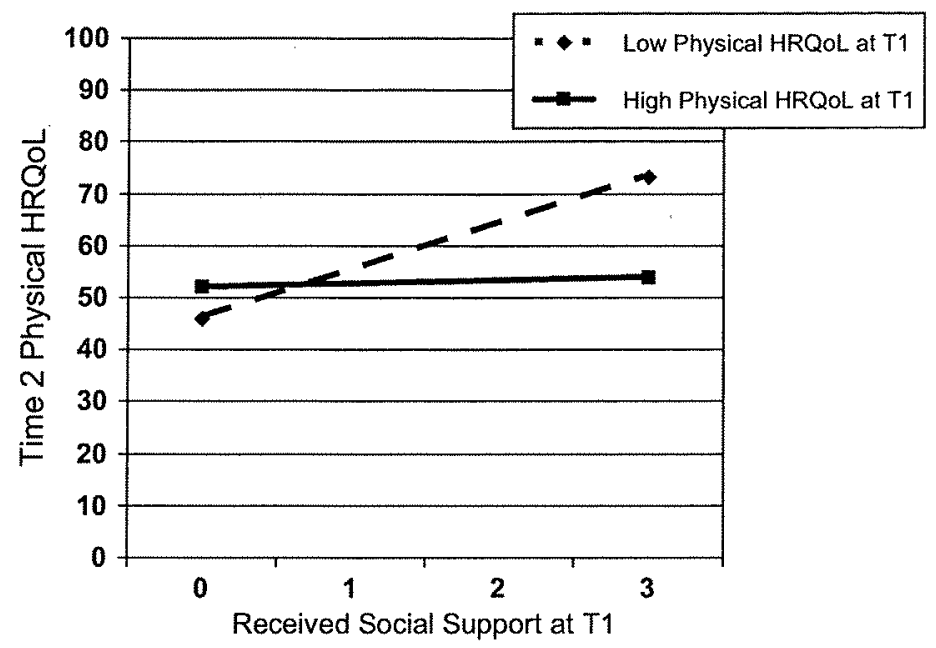

Figure 2. Interaction effect between physical HRQoL 2 weeks after surgery and patients' received social support on T2 physical HRQoL.

Note. Only the slope for low initial HRQoL reached statistical significance at $p=.05$. T1 $=$ Time 1,2 weeks after surgery; T2 =Time 2, 6 months after surgery. $\mathrm{HRQoL}=$ health-related quality of life.

Simple slope analysis demonstrated that only for low physical HRQoL at T1 did the slope prove to be different from zero, $t=3.80, p<.001$, but not for high physical HRQoL at T1, $t=.30, p=.76$. As $1 S D$ below and $1 S D$ above the centered mean as indicators for low and high physical HRQoL at Time 1 are arbitrary values, we further verified these results by testing the region of significance applying the Johnson-Neyman technique. The analysis of the region of significance showed that the values of $\mathrm{T} 1$ physical HRQoL at which the association between T1 received social support and T2 physical HRQoL was significant were lower than 1.94, $p<.05$, and higher than 142.24, $p<.05$. As minimum and maximum of the mean centered physical HRQoL was -19.55 and 18.73 , respectively, only the simple slope for the individuals who reported a T1 HRQoL lower than 1.94 was significantly different from zero. Thus, patients who had a relatively low physical HRQoL at the onset benefited from their received support in terms of physical HRQoL at T2. In contrast, for persons who reported a relatively high physical HRQoL at 2 weeks after surgery, receiving social support from their spouses was unrelated to their follow-up physical HRQoL. Overall, $36 \%$ of variance was explained in T2 physical HRQoL. Of this variance, $4 \%$ was accounted for by the interaction term.

The last regression analysis examined the effects of spouses' provided social support, physical HRQoL, mental HRQoL (all at T1), and in a second step the interaction term between spouses' provided social support and physical HRQoL on T2 physical HRQoL. Here, only earlier physical HRQoL was a significant predictor of later physical functioning. Mental HRQoL was only significant at a $10 \%$ level. Neither spouses' provided social support nor the interaction term were significant predictors of T2 physical HRQoL (see Table II). A total of $24 \%$ of variance was explained by variables in this analysis.

\section{Discussion}

The present study investigated the effects of receipt and provision of social support on HRQoL in prostate cancer patients undergoing radical prostatectomy. Moreover, it was tested whether the baseline-level of HRQoL served as a moderator of the association between received and provided social support and HRQoL 6 months later. There were no 
consistent main effects of either received social support or spousal provided social support on both outcome measures. Only received social support could positively predict change in physical HRQoL from 2 weeks to 6 months after surgery. This is partly in line with a study by Boehmer et al. (2007), who found positive effects of received social support on emotional HRQoL in cancer patients 6 months after surgery. The few resulting main effects of received social support are in contrast to the consistently positive effects of perceived social support on HRQoL reported in the literature (e.g., Helgeson, 2003). However, perceived social support reflects more a personality disposition (e.g., Sarason et al., 1986) than actual support transactions. As there is only little research on effects of received and provided social support on adjustment to grave physical illness, the investigation of both perspectives of support as predictors of HRQoL is a strength of the present study.

A methodological explanation for a lack of further simple effects of support on HRQoL might have to do with restricted range. Two weeks after surgery, both patients and their spouses reported a high intensity of support directed toward the patient. However, the positive effect of received social support found for patients who were initially worse off along with the pattern of correlations found for the entire sample yields evidence reducing this concern.

Three of the four interaction effects examined gained statistical significance and yielded the expected differential associations: Patients who had a lower mental or physical HRQoL at the beginning of the study seemed to benefit more from receiving social support. Moreover, in the case of mental HRQoL this differential effect was replicated using an indicator from a different data source, that is, spousal provided social support. The unexpected result, namely that persons who had a relatively high mental HRQoL reported a decrease in mental HRQoL over time when their spouses provided high social support, could be partly explained by examining the effects of provided social and received social support together in a multiple regression analysis. Here, the moderation effect of spousal provided social support in mental HRQoL was mostly accounted for by the patient received social support-HRQoL interaction. Although the decrease in significance of the provision by mental HRQoL interaction might be in part also due to low power, the stronger effect of the received social support by HRQoL interaction indicates that the patients' accounts are the stronger predictors.

Another explanation for the negative effect of social support provision for patients reporting a higher mental HRQoL might be that they felt overprotected by their wives. Overprotection has been shown to be detrimental to patients' well-being (e.g., Kuijer et al., 2000). One possible explanation for these negative effects is that being overprotected is in contrast to patients' need for autonomy, as evidence from a study among myocardial infarct patients suggests (Coyne, Ellard, \& Smith, 1990). Moreover, findings from a qualitative study with prostate cancer patients and their spouses also emphasized the difficulties spouses experience when dosing their support provision in order not to overprotect patients, thereby making them feel incapable of managing their own life (Gray et al., 2000). Thus, providing social support is not always beneficial for the receiver of the support. What seems to be important is the match between the provision of support and the needs of the support receiver. Optimal Matching Theory (OMT; Cutrona \& Russell, 1990) assumes that critical life events, such as cancer, pose different demands on the social support provision by important network members. The kind of social support needed (e.g., emotional, instrumental, informational) is assumed to depend on the controllability an individual experiences with regard to the stressor. According to the theory's assumptions, emotional social support is most beneficial when a specific stressor is uncontrollable, 
whereas informational/instrumental social support is of higher importance for stressors relatively under an individual's control (Cutrona, 1990). Thus, for those patients in the present study who initially reported a low HRQoL, there might have been a better match between their needs and their partners' provision than for those patients with a relatively higher initial HRQoL. Future studies might want to include subjective controllability of a stressor and different kinds of social support as suggested by OMT in order to test for potential mechanisms that explain why some individuals benefit more from their partner's social support efforts than others.

Another explanation for the negative effect of support provision for those high in early mental HRQoL might be a methodological one: For persons with very high levels of mental HRQoL at T1, a further increase in HRQoL over time might not be possible. Thus, the observed decrease in HRQoL might be explained by regression to the mean rather than by a real decrease in HRQoL. The results thus need to be interpreted with appropriate caution.

The results of the interaction effects of provision and receipt of social support and mental and physical HRQoL 2 weeks after surgery may be interpreted in the light of the Stress Buffering Hypothesis of social support (Cohen \& Wills, 1985). This theory states that people with higher levels of stress benefit more from social support than people who are only slightly stressed or who do not experience any stress at all. As our data suggest, this might also apply for people with different levels of HRQoL after a stressful life event, such as radical prostatectomy.

A limitation of the present study was that we did not have a direct measure of stress to test the Stress Buffering Hypothesis in the context of prostate cancer treatment. Nevertheless, this is the first study to shed light on the moderating effects of baseline levels of HRQoL on the association between received and provided social support with later HRQoL in the context of LRPE.

An unexpected finding was the lack of the interaction effect between earlier physical HRQoL and spouses' provided social support predicting physical HRQoL at 6 months after surgery. However, as the interaction effect between mental HRQoL at 2 weeks after surgery and provided social support could be accounted for by receipt of support, it might be that the provision of support was not the active component of the effect, but rather the subjective receipt of this spousal support provision. These findings on the importance of receiving support for mental and physical HRQoL 6 months after surgery are in contrast to findings reported by Bolger, Zuckerman, and Kessler (2000), who demonstrated that the unique effects of receiving support might sometimes even be detrimental for the recipients' well-being. In contrast, the unique effects of support provision (i.e., that the amount of support provided by a significant person is not recognized as such by the recipient and is thus invisible) are highly beneficial for the support receiver's well-being and promote adjustment to stressors.

A possible explanation for the beneficial effects of received social support that were found in the present study and that seemingly contradict the findings on invisible support by Bolger and colleagues (2000) might be that these effects are time-sensitive. The invisible support effects reported by Bolger et al. (2000) were day-to-day effects assessed by means of a diary. In the present study, a time lag of 6 months was analyzed. A study by Liang, Krause, and Bennett (2001) found that received social support strengthened the general expectation to have someone available in times of need (i.e., perceived social support). Perceived social support in turn was associated with better psychological and physical health (Sarason et al., 1990; Thompson \& Heller, 1990; Wethington \& Kessler, 1986). Thus, in the present study, receipt of support 2 weeks after surgery might have unfolded its 
effects for persons low in HRQoL by increasing their sense of perceived social support as the mediating mechanism.

A limitation of the present study was the small sample size and the relatively high attrition rate. Moreover, spouses who dropped out were significantly younger than those who continued participation. Applying MI, as was done in the present study, however, is the method of choice to account for high rates of missing values while keeping the risk of biased results at a minimum (Schafer \& Graham, 2002).

Practical implications of our study result from the finding that receiving spousal social support is especially beneficial for people with lower initial levels of HRQoL. Thus, assessing patients' HRQoL might be one method to identify those patients who are in special need of support from their spouses or relatives. Screening for such patients is also in line with suggestions based on a systematic review on the effectiveness of support groups for cancer patients (Sheard \& Maguire, 1999). Thus, if no spousal support is available for prostate cancer patients, social support groups might be a good alternative, especially for those patients who have a low HRQoL at the onset. In fact, a growing body of literature finds evidence for beneficial effects of social support interventions provided by peers or professionals on different outcomes, such as depression and self-efficacy (e.g., Weber et al., 2004). On the other hand, interventions aimed at enhancing HRQoL in prostate cancer patients could benefit from including the patients' partners. Research so far has mainly focused on peer or professional support interventions for patients (e.g., Hoey, Ieropoli, White, \& Jefford, 2008; Weber et al., 2004). There are also studies demonstrating beneficial effects of social support groups for the wives of prostate cancer patients on their well-being (e.g., Manne, Babb, Pinover, Horwitz, \& Ebbert, 2004). In light of the results of the present study taking on a dyadic perspective, that is, developing support groups for both patients and their spouses together, appears to be a worthwhile next step.

\section{References}

Aiken, L. S., \& West, S. G. (1991). Multiple regression: Testing and interpreting interactions. Newbury Park, CA: Sage. Antonucci, T. C. (2001). Social relations: An examination of social networks, social support, and sense of control. In J. E. Birren, \& K. W. Schaie (Eds.), Handbook of the psychology of aging (5th edn) (pp. 427-453). San Diego, CA: Academic Press.

Barnard, J., Rubin, D. B., \& Schenker, N. (2001). Multiple imputation. In N. J. Smelser, \& P. B. Baltes (Eds.), International encyclopedia of the social and behavioral sciences (Vol. 20 pp. 10204-10210). Oxford, UK: Elsevier Science.

Basillote, J. B., Ahlering, T. E., Skarecky, D. W., Lee, D. I., \& Clayman, R. V. (2004). Laparoscopic radical prostatectomy: Review and assessment of an emerging technique. Surgical Endoscopy, 18, 1694-1711.

Boehmer, S., Luszczynska, A., \& Schwarzer, R. (2007). Coping and quality of life after tumor surgery: Personal and social resources promote different domains of quality of life. Anxiety, Stress, E Coping, 20, 61-75.

Bolger, N., Zuckerman, A., \& Kessler, R. C. (2000). Invisible support and adjustment to stress. Fournal of Personality and Social Psychology, 79, 953-961.

Bowling, A. (1991). Measuring health: A review of quality of life measurement scales. Buckingham, UK: Open University Press.

Braslis, K. G., Santa-Cruz, C., Brickman, A. L., \& Soloway, M. S. (1995). Quality of life 12 months after radical prostatectomy. British fournal of Urology, 75, 48-53.

Bullinger, M., \& Kirchberger, J. (1998). Der SF-12. [The SF-12.] In M. Bullinger \& T. J. Kirchberger (Eds), Der SF-36-Fragebogen zum Gesundheitszustand [The SF-36 Health Survey] (pp. 65-72). Göttingen, Germany: Hogrefe.

Campbell, L. C., Keefe, F. J., McKee, D. C., Edwards, C. L., Herman, S. H., Johnson, L. E., et al. (2004). Prostate cancer in African Americans: Relationship of patient and partner self-efficacy to quality of life. Fournal of Pain and Symptom Management, 28, 433-444.

Cohen, S., \& Wills, T. A. (1985). Stress, social support, and the buffering hypothesis. Psychological Bulletin, 98, 310-357. 
Coombs, R. H. (1991). Marital status and personal well-being: A literature review. Family Relations, 40, $97-102$.

Coyne, J. C., Ellard, J. H., \& Smith, D. A. (1990). Social support, interdependence, and the dilemmas of helping. In B. R. Sarason, I. G. Sarason, \& G. R. Pierce (Eds.), Social support: An interactional view (pp. 129-149). Oxford, UK: Wiley.

Cutrona, C. E. (1990). Stress and social support: In search of optimal matching. Fournal of Social and Clinical Psychology, 9, 3-14.

Cutrona, C. E., \& Russell, D. W. (1990). Type of social support and specific stress: Toward a theory of optimal matching. In B. R. Sarason, I. G. Sarason, \& G. R. Pierce (Eds.), Social support: An interactional view (pp. 319 366). New York: Wiley.

Eton, D. T., \& Lepore, S. J. (2002). Prostate cancer and health-related quality of life: A review of the literature. Psycho-Oncology, 11, 307-326.

Eton, D. T., Lepore, S. J., \& Helgeson, V. S. (2005). Psychological distress in spouses of men treated for early-stage prostate carcinoma. Cancer, 103, 2412-2418.

German Federal Statistical Office (2006). Sterbefälle nach den 10 häufigsten Todesursachen [Number of deaths sorted by the ten most common causes of death]. Retrieved 7 June 2006, from http://www.destatis.de/basis/d/ gesu/gesutab20.php\#nicht

Gray, R. E., Fitch, M., Phillips, C., Labreque, M., \& Fergus, K. (2000). Managing the impact of illness: The experiences of men with prostate cancer and their spouses. Fournal of Health Psychology, 5, 531-548.

Helgeson, V. S. (2003). Social support and quality of life. Quality of Life Research, 12(Suppl. 1), 25-31.

Hoey, L. M., Ieropoli, S. C., White, V. M., \& Jefford, M. (2008). Systematic review of peer-support programs for people with cancer. Patient Education Counseling, 70, 315-337.

Jenkinson, C., Layte, R., Jenkinson, D., Lawrence, K., Petersen, S., Paice, C., et al. (1997). A shorter form health survey: Can the SF-12 replicate results from the SF-36 in longitudinal studies? Fournal of Public Health Medicine, $19,179-186$.

Knoll, N., Burkert, S., Rosemeier, H. P., Roigas, J., \& Gralla, O. (2007). Predictors of spouses' provided support for patients receiving laparoscopic radical prostatectomy peri-surgery. Psycho-Oncology, 16, 312-319.

Koopman, C., Hermanson, K., Diamond, S., Angell, K., \& Spiegel, D. (1998). Social support, life stress, pain and emotional adjustment to advanced breast cancer. Psycho-Oncology, 7, 101-111.

Kuijer, R. G., Ybema, J. F., Buunk, B. P., De Jong, G. M., Thijs-Boer, G., \& Sanderman, R. (2000). Active engagement, protective buffering, and overprotection: Three ways of giving support by intimate partners of patients with cancer, fournal of Social and Clinical Psychology, 19, 256-275.

Liang, J., Krause, N. M., \& Bennett, J. M. (2001). Social exchange and well-being: Is giving better than receiving? Psychology and Aging, 16, 511-523.

Little, R. J. A., \& Rubin, D. B. (2002). Statistical analysis with missing data (2nd edn). Hoboken, NJ: Wiley.

Litwin, M. S., McGuigan, K. A., Shpall, A. I., \& Dhanani, N. (1999). Recovery of health related quality of life in the year after radical prostatectomy: Early experience. Fournal of Urology, 161, 515-519.

Lubeck, D. P., Litwin, M. S., Henning, J. M., Stoddard, M. L., Flanders, S. C., \& Carroll, P. R. (1999). Changes in health-related quality of life in the first year after treatment for prostate cancer: Results from CaPSURE. Urology, 53, 180-186.

Maliski, S. L., Heilemann, M. V., \& McCorkle, R. (2002). From "death sentence" to "good cancer": Couples" transformation of a prostate cancer diagnosis. Nursing Research, 51, 391-397.

Manne, S., Babb, J., Pinover, W., Horwitz, E., \& Ebbert, J. (2004). Psychoeducational group intervention for wives of men with prostate cancer. Psycho-Oncology, 13, 37-46.

Muthén, L. K., \& Muthén, B. O. (1998). Mplus user's guide (4th edn). Los Angeles, CA: Muthén \& Muthén.

Parker, P. A., Baile, W. F., DeMoor, C., \& Cohen, L. (2003). Psychosocial and demographic predictors of quality of life in a large sample of cancer patients. Psycho-Oncology, 12, 183-193.

Preacher, K. J., Curran, P. J., \& Bauer, D. J. (2006). Computational tools for probing interaction effects in multiple linear regression, multilevel modeling, and latent curve analysis. Fournal of Educational and Behavioral Statistics, $31,437-448$

Ptacek, J. T., Pierce, G. R., Ptacek, J. J., \& Nogel, C. (1999). Stress and coping processes in men with prostate cancer: The divergent views of husbands and wives. Fournal of Social and Clinical Psychology, 18, $299-324$.

Ries, L. A. G., Harkins, D., Krapcho, M., Mariotto, A., Miller, B. A., Feuer, E. J, et al. (Eds). (2006). SEER Cancer Statistics Review, 1975-2003, National Cancer Institute. Bethesda, MD, http://seer.cancer.gov/csr/ 1975_2003/, based on November 2005 SEER data submission, posted to the SEER web site 2006.

Roberts, K. J., Lepore, S. J., \& Helgeson, V. (2006). Social-cognitive correlates of adjustment to prostate cancer. Psycho-Oncology, 15, 183-192. 
Roumeguere, T., Bollens, R., Vanden Bossche, M., Rochet, D., Bialek, D., Hoffman, P., et al. (2003). Radical prostatectomy: A prospective comparison of oncological and functional results between open and laparoscopic approaches. World fournal of Urology, 20, 360-366.

Rubin, D. B. (1987). Multiple imputation for nonresponse in surveys. New York: Wiley.

Sarason, B. R., Pierce, G. R., \& Sarason, I. G. (1990). Social support: The sense of acceptance and the role of relationships. In B. R. Sarason, I. G. Sarason, \& G. R. Pierce (Eds.), Social support: An interactional view (pp. 97-128). Oxford, UK: Wiley.

Sarason, I. G., Sarason, B. R., \& Shearin, E. N. (1986). Social support as an individual difference variable: Its stability, origins, and relational aspects. Fournal of Personality and Social Psychology, 50, 845-855.

Schafer, J. L. (1999). NORM: Multiple imputation of incomplete multivariate data under a normal model, version 2. Software for Windows 95/98/NT. Retrieved 2 January 2003, from http://www.stat.psu.edu/ jls/misoftwa.html

Schafer, J. L., \& Graham, J. W. (2002). Missing data: Our view of the state of the art. Psychological Methods, 7, 147177.

Schafer, J. L., \& Olsen, M. K. (1998). Multiple imputation for multivariate missing-data problems: A data analyst's perspective. Multivariate Behavioral Research, 33, 545-571.

Schulz, U., \& Schwarzer, R. (2003). Soziale Unterstützung bei der Krankheitsbewältigung: Die Berliner Social Support Skalen (BSSS) [Social support in coping with illness. The Berlin Social Support Scales (BSSS)]. Diagnostica, 49, 73-82.

Schulz, U., \& Schwarzer, R. (2004). Long-term effects of spousal support on coping with cancer after surgery. Fournal of Social and Clinical Psychology, 23, 716-732.

Schwarzer, R., Knoll, N., \& Rieckmann, N. (2003). Social support. In A. Kaptein, \& J. Weinman (Eds.), Introduction to health psychology (pp. 158-182). Oxford, UK: Blackwell.

Sheard, T., \& Maguire, P. (1999). The effect of psychological interventions on anxiety and depression in cancer patients: Results of two meta-analyses. British fournal of Cancer, 80, 1770-1780.

Thompson, M. G., \& Heller, K. (1990). Facets of support related to well-being: Quantitative social isolation and perceived family support in a sample of elderly women. Psychology and Aging, 5, 535-544.

Türk, I., Deger, S., Winkelmann, B., Schönberger, B., \& Loening, S. A. (2001). Laparoscopic radical prostatectomy. Technical aspects and experience with 125 cases. European Urology, 40, 46-52.

Visser, A., van Andel, G., Willems, P., Voogt, E., Dijdstra, A., Rovers, P., et al. (2003). Changes in health-related quality of life of men with prostate cancer 3 months after diagnosis: The role of psychosocial factors and comparisment with benign prostate hyperplasia patients. Patient Education and Counseling, 49, 225-232.

Weber, B. A., Roberts, B. L., Resnick, M., Deimling, G., Zauszniewski, J. A., Musil, C., et al. (2004). The effect of dyadic intervention on self-efficacy, social support, and depression for men with prostate cancer. PsychoOncology, 13, 47-60.

Wethington, E., \& Kessler, R. C. (1986). Perceived support, received support, and adjustment to stressful life events. Fournal of Health and Social Behavior, 27, 78-89. 